\title{
Current Topics Concerning Joint Preservation and Minimally Invasive Surgery of the Hip
}

\author{
Editorial Comment
}

\author{
Edwin P. Su, MD
}

Received: 18 July 2012/Accepted: 1 August 2012

(C) Hospital for Special Surgery 2012

Our understanding of the hip over the last 20 years has evolved from that of a simple ball and socket joint, to that of a complex interplay between ligaments, bony anatomy, cartilage, and labrum working in 6 degrees of freedom. With our increased understanding of the normal anatomy and pathologic mechanisms of damage to these structures has come an improved ability to diagnose problems, and subsequently offer surgical treatments.

One of the fastest-growing fields in orthopedic surgery is that of the hip specialist. Because of the wide range of ages and conditions that can afflict the hip, many academic centers have developed "hip centers", where several physicians provide a continuum of care for hip conditions. At Hospital for Special Surgery, the Center for Hip Preservation was established in 2010, with the goal of providing care for patients of all ages with hip pathology, ranging from developmental dysplasia of the hip, to sports-related injuries, to degenerative arthritis. Physiatrists, pediatric orthopedic surgeons, sports medicine surgeons, and arthroplasty surgeons all practice in the center, allowing for a spectrum of nonoperative and operative treatments.

This increased understanding of the hip could not have come without pioneering educators such as Paul Beaule, MD, who embraced progressive treatments for hip pathology such as periacetabular osteotomy, surgical hip dislocation, and hip resurfacing. From his interest in the pathologic mechanisms of the hip and treatments for such, in 2001 Dr. Beaule developed a course focused upon "Joint Preserving and Minimally Invasive Surgery of the Hip". Through the years, the course has evolved to include not only acetabular osteotomy, but also hip arthroscopy, open

\footnotetext{
E. P. $\mathrm{Su}, \mathrm{MD}(\triangle)$

Hospital for Special Surgery,

535 East 70th Street,

New York, NY 10021, USA

e-mail: sue@hss.edu

E. P. $\mathrm{Su}, \mathrm{MD}$

Clinical Orthopaedic Surgery, Weill Cornell Medical College,

New York, NY 10065, USA
}

hip dislocation, and primary and revision hip arthroplasty. This edition of the HSS Journal contains the proceedings of the 9th course on Joint Preserving and Minimally Invasive Surgery of the Hip, held in New York City in 2011.

This collection of papers represents a diverse experience with a wide range of hip conditions and surgical treatments. In the hip preservation arena, Drs. Tibor and Leunig review the pros and cons of labral resection and repair during FAI treatment; $\mathrm{Mr}$. Ng and Dr. Beaule use finite element analysis to examine the mechanical loading effects of a cam during squat and stand; Drs. Boone and Sierra examine the results of FAI treatment in patients over the age of 40. Primary hip arthroplasty topics include the discussion of metal-on-metal bearings by Dr. Migaud and Girard; Drs. Su and Walter debate the merits of a ceramic-on-ceramic THA; Drs. Beaule describes the phenomenon of groin pain after hip resurfacing; and Dr. Amstutz gives his 40 year perspective on hip resurfacing. Finally, Drs. Mertl and Migaud discuss the treatment of recurrent THA dislocation with a dual mobility bearing; and Drs. Konan and Haddad discuss whether or not current hip scores are sufficiently sensitive and robust, or whether we need the development of a new, more discriminating hip score.

These papers memorialize the educational material presented at the course and provide a snapshot into our experience with hip disorders circa 2011. They will undoubtedly advance our understanding of the pathologic mechanisms of the hip joint and help us to refine our surgical treatments. The 10th course on Joint Preserving and Minimally Invasive Surgery of the Hip, to be held in Ottawa on May 30 to June 1, 2013, is sure to further our comprehension of the hip joint. It is an exciting time to be a hip specialist!

Disclosure Each author certifies that he or she has no commercial associations (e.g., consultancies, stock ownership, equity interest, patent/licensing arrangements, etc.) that might pose a conflict of interest in connection with the submitted article. 